\title{
TRANSFORMACIONES EDUCACIONALES EN EL SIGLO XXI, CAMBIOS EN EL MODO DE ACTUACIÓN PROFESIONAL
}

\author{
EDUCATIONAL TRANSFORMATIONS IN THE XXI CENTURY, \\ CHANGES IN PERFORMANCE MODE
}

\section{RENÉ MANUEL VELÁZOUEZ ÁVILA}

Facultad de Educación a Distancia, Universidad de La Habana, Cuba.

rvelazquez@fed.uh.cu

RESUMEN

En aras de la transoformaciones que emanan como producto del perfeccionamiento continuo del proceso docente educativo en la Universidad del Siglo XXI, se ofrece un ejercicio epistemológico crítico de la racionalidad y consistencia de diferentes concepciones que sobre el tema, objeto de estudio de esta propuesta, se debaten en la contemporaneidad educativa. Se procede al desentrañamiento teórico-metodológico del Modo de Actuación Profesional en sus prospecciones conceptuales y fenoménicas, elementos estos que sentaron las bases para una propensión coherente de definición por parte del autor, así como también, ofrecer elementos que condicionan las transformaciones en las que opera el perfeccionamiento del modo de actuación profesional desde una perspectiva interdisciplinar.

PALABRAS CLAVE: modo actuación profesional, perfeccionamiento, transformaciones educacionales.
For the sake of transformations that emanate as a result of continuous improvement of the educational process at the University of the XXI Century, offered a critical epistemological exercise of rationality and consistency of different views on the topic: debate in educational contemporaneity, that is study of this proposal. It proceeds to the theoretical and methodological disembowelment of professional performance in its conceptual and phenomenal prospections, elements that set the stage for a propensity consistent definition by the author, as well as, provide elements that condition transformations in which it operates the improvement of professional performance from an interdisciplinary perspective.

KEYWORDS: performance mode, improvement, educational transformations 
INTRODUCCIÓN

El contexto en que se sitúan los centros de Educación Superior es cambiante, el uso de las tecnologías de la información y las comunicaciones (TIC), ha transformado la manera de pensar y la forma de actuar en la búsqueda y gestión del aprendizaje. Si bien es cierto, dirigir el proceso docente educativo en las condiciones actuales de la educación en el siglo XXI, exige de un profesional más competente y capacitado para asimilar todas las transformaciones que ocurren desde el punto de vista social, económico, cultural y desde las ciencias propiamente expresado. Sin duda alguna, pensar en un profesional que dirija el aprendizaje con métodos tradicionales, es condicionar desmotivación en los estudiantes, que lo limita como futuros profesionales.

Sin lugar a dudas, este proceso de transformación ha sido complejo, por lo que ha sido necesario la participación activa de profesionales capaces de enfrentar los cambios educativos, y formarse modos de actuación que les permitan hacer valoraciones críticas de la realidad, de su preparación y de las posibilidades de transformación, dirigidas a concebir de manera diferente su actuación en el seno de la escuela. En este sentido, podría preguntarse: ¿qué hacer para transformar el modo de actuación de los profesionales en las condiciones actuales?, ¿qué elementos identifican su transformación?, ¿cuáles herramientas teóricas y metodológicas pueden utilizarse?

A nivel ministerial, se aborda el tema de cambios en el modo de actuación de los directivos, esto es asimilado como una política necesaria para enfrentar tareas diversas, de manera que pueda incidir positivamente en sus subordinados. El análisis desde la educación es similar, solo que el tratamiento es más complejo, precisamente abordar la temática con docentes, encargados de la dirección de un proceso de educación, formación y aprendizaje, resulta más complejo, se trata de la revisión de métodos, estilos de aprendizaje, dominios de contenidos, entre otros elementos que tipifican, desde la valoración de este autor, la transformación del actual del profesional que enfrenta la difícil y hermosa tarea de educar desde el aula.

Los continuos errores dentro del proceso de enseñanza-aprendizaje, los reclamos de los educandos, resultados en evaluaciones e informes presentados en controles desarrollados por las direcciones de educación a nivel municipales y provinciales, demuestra que existen barreras que limitan el desarrollo de una dinámica que diversifique la manera de conducir el trabajo del docente desde una perspectiva integradora.

En la propuesta que se presenta se ofrecen reflexiones importantes sobre modo de actuación desde una perspectiva interdisciplinar, se propone una definición como parte de la experiencia del investigador y de otros colaboradores que participan de los resultados, además se presentan una serie de elementos necesarios a tener en cuenta para el perfeccionamiento del modo de actuación interdisciplinario del profesional de la educación.

\section{DESARROLLO}

El término modo de actuación del docente ha sido utilizado frecuentemente en la literatura relacionada con formación de profesores, al referirse no solo a la formación inicial, también a la formación permanente. López A., E. (2000) plantea que el modo de actuación del docente se enmarca en tres campos diferentes, pero estrechamente relacionados: como director del PDE, como investigador del PDE y como activista social.

Al delimitar el contenido fundamental de cada uno de estos campos, se revela que el modo de actuación del docente está dado por la forma que éste adopta para desempeñarse como rector del proceso, y para ello necesitará de la comprensión teórica de lo que va a realizar como máximo responsable, entiéndase entonces, que precisará tener gran afecto por la tarea que desempeña, poseer los conocimientos necesarios que le permitan acceder a cada una de estas, y en correspondencia con esto, dependerá la forma en que se manifieste su comportamiento.

Para comprender el significado de modo de actuación, es necesario partir del análisis de la literatura fundamental que así lo refiere, $<$ modo de actuación del docente>, se entiende como la forma históricamente condicionada de desempeñarse el docente, constituido por el conjunto de procedimientos, métodos y estados para la comunicación y la actividad pedagógica, los cuales revelan un determinado nivel de desarrollo de sus habilidades y capacidades, así como de constructos, rutinas, esquemas y modelos de actuación profesional. (García, 1997)

De la valoración de esta definición, es importante plantear que los elementos que la forman permiten comprender su significado en el análisis de la formación interdisciplinaria del docente, atendiendo a que en esta concepción teórica 
se hace referencia al papel del mismo como rector del proceso, así como de las vías y medios con los que cuenta para desempeñarse con un determinado nivel de desarrollo profesional. De igual forma, se revelan dos componentes que son fundamentales para perfeccionar el modo de actuación del profesional, un componente estructural donde se manifiestan los sustentos teóricos que nutren la plataforma del modo de actuación, y que condicionan la estructura interna que este adopta; un componente funcional que constituye la parte dinámica del modo de actuación y se manifiesta a través de la actividad del profesor durante su ejercicio profesional.

Estos componentes constituyen un sistema dentro de la actividad pedagógica que desarrolla el docente en el proceso de perfeccionar su modo de actuación. Un lugar destacado en este proceso lo ocupan los métodos y los procedimientos que se emplean para el análisis y la discusión del quehacer docente, lo que constituye el elemento clave para la transformación y perfeccionamiento de la actividad docente, propiciando que se tome conciencia de la importancia de su actuación como sujeto. Significa que "...tomar conciencia del método con que se opera, es ver cómo éste realmente tiene lugar, qué funciona en la actividad, cuál es la conducta ante los discípulos, colegas y grupos, cuáles son las representaciones sobre las que operan los métodos y cómo pueden ser estudiados" (García, 1997).

Cuando el docente asimila de manera consciente los métodos con que opera, cuando descubre cómo realmente estos tienen lugar, y cómo funcionan en la actividad, entonces, está perfeccionando su modo de actuación, lo que le permite trabajar sistemáticamente con un volumen mayor de información de manera integrada.

En el procedo docente-educativo (PDE), perfeccionar el modo de actuación desde una óptica interdisciplinaria implicará establecer en la práctica, una comunicación dialógica con el claustro, de manera que logren proyectar acciones encaminadas a la búsqueda del conocimiento integrado, indagando la forma en que se experimente motivación por la actividad, que se adquiera conocimiento y a su vez se establezca un pensamiento crítico y reflexivo que garantice la modificación de una determinada posición, con criterios y convicciones personales, lo que contribuirá a una nueva forma de manifestación de comportamiento en el individuo.
En la formación inicial de los docentes, el modo de actuación del profesor encargado de llevar a cabo este proceso ejerce una gran influencia, no sólo por los objetivos que trata de alcanzar y los contenidos que desarrolla, sino también, por los procedimientos, métodos y medios que utiliza para guiar su actividad formativa. En este sentido, autores como Hernández (1998) y Garcés (1999), reconocen y aceptan el efecto reproductor o de modelaje que ejerce el modo de actuación de los docentes que encauzan el proceso de formación educacional. De esta forma se asume la idea de conferirle un papel significativo a la disposición, interés y desempeño que muestre el docente en el desarrollo del PEA, para lograr un trabajo que permita perfeccionar su modo de actuación desde una óptica interdisciplinaria.

Por otra parte, el desarrollo de la investigación constituye una manifestación evidente y preponderante de la transformación del modo de actuación de los profesionales, como resultado del avance científico (Ortiz, 2011).

En correspondencia con los criterios abordados, y de acuerdo a las necesidades didácticas de su proyección, el autor define como Modo de Actuación Interdisciplinario: La forma en que el docente manifiesta comportamientos congruentes con la dirección de acciones y procedimientos formativos orientados a la concreción didáctica de la interdisciplinariedad, sobre la base del dominio teórico- metodológico de los contenidos, que revela el desarrollo de sus habilidades y capacidades en torno a las relaciones instructivas, educativas y desarrolladoras del conjunto de disciplinas que conforman el currículo escolar.

Algunas de las condiciones que debe poseer un docente para poseer un modo de actuación interdisciplinario son:

1. Concebir la intervención docente a partir de la relación proceso-producto. El profesor, en todo su accionar, requiere pensar en las transformaciones que habrán de realizarse; pero partiendo del análisis de la repercusión individual de estos resultados, en correspondencia con los criterios de formación que orientan la actividad educativa.

2. Adquirir una conciencia acerca de lo que se quiere hacer y lograr, o sea, de los modos de actuación a que se aspira y de sus representaciones en el PEA.

3. Denotar dominio teórico-práctico de su labor, y ser capaz de contextualizar la 
misma en los diferentes niveles donde esta se proyecta y concreta.

4. Estimular la realización de las actividades del colectivo pedagógico en equipos interdisciplinarios, lo que significa que la actividad docente debe asumirse desde las perspectivas de una concepción interdisciplinaria.

5. Asumir la formación de los estudiantes con el goce y la responsabilidad que amerita esta tarea. Tal cuestión será un elemento importante para estrechar el lazo entre las dimensiones motivacional-afectiva y cognitiva-instrumental de la actividad docente.

La consolidación de esta forma de trabajo en los docentes, contribuirá sin duda alguna al perfeccionamiento del modo de actuación vigente, fomentando la activación de actitudes de trabajo interdisciplinario entre los profesionales, ampliando la cultura y la actualización didáctica para estimular un clima de creatividad entre estos, así como también perfeccionar el control y la evaluación del desempeño del docente.

Carácter Interdisciplinario del Modo de Actuación Profesional.

La posibilidad de lograr perfeccionar el modo de actuación de los docentes en las instituciones escolares, implica una transformación profunda en la manera de pensar, aprender, comportarse y en los métodos de enseñanza que utiliza, por lo que será necesario perfeccionarlos, unido a las nuevas modificaciones en este tipo de educación.

La confrontación teórica en el campo de las transformaciones en el modo de actuación del docente, lleva a comprender que la interdisciplinariedad aparece como un principio en los profesionales de la educación que contribuirá al perfeccionamiento de su actividad pedagógica, lo que significa, como ha dicho Perera Curmena (2001) “...permite la dirección del proceso de enseñanza-aprendizaje que involucra y compromete a los sujetos en la apropiación activa de conocimientos, habilidades y valores a través del establecimiento de vínculos interdisciplinares", sin lugar a dudas, esto se hará con el objetivo de formar profesionales capacitados para resolver integralmente, los problemas que surjan en la práctica, y con dominio del contenido para garantizar la autopreparación y la actualización continuada de saberes en sus discípulos."
En este espacio se sostiene el criterio de que el estudio del modo de actuación viene dado por dos aspectos fundamentales, el afectivomotivacional y el cognitivo- instrumental, para determinar los comportamientos de las personas hacia las distintas actividades que se desean realizar. Son procesos que se aprenden y se autorregulan como resultado de la actividad del individuo; pues no se puede concebir la actividad al margen de la participación consciente, de la interacción consciente y organizada del docente con el alumno y viceversa.

En ocasiones, se observan docentes que dirigen la actividad de aprendizaje de forma pasiva, se convierten en repartidores de información sin argumentos que despierte la motivación del estudiantes por la búsqueda, el desarrollo del pensamiento interdisciplinar, la interdisciplinariedad aparece como una actividad frontal de poca importancia y duración, no tiende al análisis y valoración de los principales elementos del conocimiento que pueden explicar la esencia de las disciplinas y sus asignaturas de manera más sencilla y entender los principales problemas que contribuyen a la formación de los estudiantes.

Lo deseable y científicamente fundamentado conllevaría al establecimiento de actividades que interrelacionen armónicamente lo cognitivo con lo afectivo para generar cambios profundos en el modo de actuación de los docentes y que estos se manifiesten de forma racional frente a los cambios.

El aspecto afectivo tiene mayor importancia en la transformación del individuo, pues las vivencias afectivas promueven mejor los cambios en el comportamiento, debido a que el sujeto experimenta sensaciones que lo conducen a mostrarse de una manera o de otra. (Fishbein y Azjen, citado por Núñez Coba, 2003), De esta apreciación se puede extraer una importante reflexión y es que si hoy no se han alcanzado grandes éxitos en el trabajo interdisciplinario, es precisamente porque no se ha logrado poner a los sujetos en contacto directo con el análisis de la realidad, y para el conocimiento de esa realidad, una forma de hacerlo es a partir de lograr que el docente interiorice, haga suyo los elementos del conocimiento que permiten establecer nexos interdisciplinares correspondientes a las asignaturas desde diversas perspectivas. Sin duda alguna, esto sería posible si los docentes trabajaran con un modo de actuación interdisciplinario. 
El proceso de transformación del modo de actuación se expresa de una forma $u$ otra en una disposición de la persona hacia la actividad que desarrollará, lo que significa que es resultado de los procesos cognitivo y afectivo, puesto que cada uno adopta lo específico para dirigir la conducta humana.

Los procesos afectivos tienen entre sus funciones, expresar cómo afecta los objetos y situaciones de la realidad a las necesidades de los individuos, y en consecuencia modificar la esfera afectiva del ser humano. Cuando el docente siente afecto por lo que realiza, implica a su vez que está generándose un proceso de motivación, categoría estrechamente relacionada, de ahí que sea necesario comprender que esta categoría funciona en una unidad dialéctica a trabajar dentro del proceso de perfeccionamiento del modo de actuación.

\section{MOTIVACIÓN Y MODO DE ACTUACIÓN PROFESIONAL}

La motivación es la causa del comportamiento de un organismo, o razón por la que un organismo lleva a cabo una actividad determinada. González Serra (1995), se refiere al conjunto concatenado de procesos psíquicos (que implican la actividad nerviosa superior y reflejan la validez objetiva a través de las condiciones internas de la personalidad) que conteniendo el papel activo y relativamente autónomo de la personalidad, y en constante transformación y determinación recíprocas con la actividad externa, sus objetivos y estímulos, van dirigidos a satisfacer las necesidades del hombre y en consecuencia, regulan la dirección (el objeto-meta) y la intensidad o activación del comportamiento, manifestándose como actividad motivada,

No obstante, en un aspecto donde suelen coincidir casi todos los estudiosos de la motivación, independientemente de la posición teórica que asuman, es el de que las motivaciones están asociadas al acto creador, por lo que pueden y deben ser estudiadas mediante el despliegue de diversas acciones educativas, durante el PEA.

Comprender la relación existente entre motivación y aprendizaje, se hace pertinente a partir de la categoría de aprendizaje desde una perspectiva histórico-cultural, que lo asume como un proceso esencial de construcción y reconstrucción de conocimientos, formas de comportamiento, actitudes, valores, modo de actuación y afectos, que se producen en condiciones de interacción social en un medio sociohistórico concreto, en dependencia del nivel de conocimientos que posee el sujeto, de sus intereses, estados de ánimo, actitudes y valores hacia diferentes esferas de la realidad social y personal; que lo conducen a su desarrollo personal y, en ocasiones, también a los sujetos con los cuales interactúa. Desde esta posición, la enseñanza se analiza como fuente del desarrollo y la actividad concreta que el sujeto realiza como su determinante. En éste sentido, el análisis debe conducirse hasta el examen de la unidad primaria de la actividad, la acción que el sujeto realiza.

En esta concepción se comprende que si el conocimiento que se va construyendo se despliega en un ambiente gozoso, realmente estamos desarrollando una fuerza interior que tiene que ver con lo que es la motivación y su papel en el proceso de aprendizaje de los alumnos.

González Serra (1995) considera que, en la motivación están presentes factores extrínsecos y factores intrínsecos. Se ha definido a la motivación intrínseca como aquella que conduce a la realización del comportamiento aunque no existan premios o cualquier otro tipo de satisfacciones externas, o como aquella motivación interna referida a unos cometidos en que la ejecución del proceso creativo y la satisfacción que en él se alcanza constituyen en sí mismo la meta. La motivación extrínseca es la que resulta de los reforzadores externos o aquella donde el producto creativo es sólo el medio para obtener determinados objetivos o realizaciones externas, donde la satisfacción proviene de los fenómenos sociales concomitantes.

Es precisamente a través de la motivación, que el hombre se entrelaza con la realidad concreta, es la motivación un tipo de proceso mediador o reflejo del ambiente, en el cual el carácter social e históricamente determinado de los objetos y fenómenos que refleja, influye y condiciona sus características estructurales y modos de funcionar.

A partir de las diferentes aportaciones al estudio científico de la motivación, se ha logrado una concepción integradora de la motivación, que implica que ésta conforma el sistema de motivos que funciona en la actividad, dirigida a satisfacer las necesidades del hombre y, en consecuencia, regula la dirección y la intensidad del comportamiento del individuo ante determinadas situaciones.

El motivo estimula al hombre a actuar, los motivos reflejan una necesidad humana, se puede decir que el motivo constituye la objetivación de la necesidad (se convierte en motivo cuando se organiza la conducta). Las necesi- 
dades del individuo son infinitas, por eso, si el estudiante no quiere estudiar, si no siente la necesidad de estudiar, no va a aprender, pues su esfera motivacional probablemente esté puesta en el amor, el deporte u otra actividad social e individual.

El estudio no se convierte en actividad cuando el mismo no satisface la necesidad del estudiante, lo que implica su inexistencia como motivo, por ejemplo, si quiere obtener el título para alcanzar prestigio, el estudio es más bien una acción pero no una actividad. Para el PEA, la mejor variante se da cuando los estudiantes encuentran en el estudio un verdadero motivo. La motivación, en tal sentido, debe ser entendida como un proceso resultante de la unidad de lo cognitivo y lo afectivo.

Desde las perspectivas analizadas subyace una pregunta: ¿Cómo estimular el desarrollo de la motivación en el PEA? Algunas recomendaciones tendientes a la contestación de esta interrogante pueden sintetizarse en las ideas siguientes:

- Utilización de la enseñanza problémica como generatriz del conocimiento.

- El vínculo con las necesidades cognoscitivas y exigencias del desarrollo social, para subrayar la utilidad y el significado del conocimiento en el contexto en el que se desenvuelve el individuo.

- Participación del individuo en la definición de algunos objetivos y contenidos de su interés.

- Dosificar las tareas y exigencias para contribuir a fortalecer el interés de todos los implicados en la actividad.

- Considerar el error o las insuficiencias como elementos que contribuyan al desarrollo personal de los sujetos.

- Tratar de lograr un mayor interés del individuo por el proceso de conocimiento y no tan sólo por sus resultados.

En este mismo sentido, es preciso tener en cuenta otros elementos de evidente incidencia en el tratamiento formativo de la motivación, entre éstos:

- La necesidad de que el profesor vaya ubicando cuáles son las resistencias que se manifiestan en sus estudiantes.

- Es necesaria la demostración efectiva de la calidad profesional del docente y de sus fortalezas cognoscitivas y metodológicas.
- Se hace imprescindible manifestar flexibilidad en el manejo de los contenidos, tomando en cuenta las características del grado y el grupo.

- Se requiere una adecuada utilización del amor propio de cada persona para aprovechar positivamente sus potencialidades cognitivas e instrumentales, ya que en algunas ocasiones tienden a decir no puedo por no decir tengo miedo.

- Reafirmar el sentimiento de autoconfianza y elevar la autoestima de los alumnos.

En sentido general, es válido señalar que la motivación es una fuerza expansiva y contagiosa, que le permite a los profesores penetrar en la esencia de lo que estudia, es también un proceso que se construye, que permite que se adquiera mayor confianza en lo que se hace, por lo que como profesores precisamos de su asunción como exigencia y prioridad para contribuir al perfeccionamiento del modo de actuación interdisciplinario de los docentes.

En consecuencia con lo antes expuesto se ofrecen algunas condiciones que tributan al perfeccionamiento del modo de actuación interdisciplinario en los docentes.

- Poseer dominio integral de los elementos del conocimiento de su disciplina en plena correspondencia con las otras que forman el currículo.

- Determinar nexos interdisciplinarios que pueden establecerse como premisa indispensable que garantice el desarrollo del trabajo de las disciplinas en la carrera.

- Asumir el estudio de los contenidos de las disciplinas, a partir de la propuesta de situaciones de aprendizaje o experiencias cotidianas de los estudiantes.

- Concebir la actividad metodológica desde una óptica interdisciplinaria, a partir de la definición de líneas integradoras de trabajo metodológico que motiven al docente al conocimiento holístico de todas las disciplinas.

- Emplear métodos y procedimientos activos y novedosos que permitan la concreción formativa de la actividad metodológica de manera interdisciplinaria.

- Concebir la actividad investigativa como una vía fundamental que fortalece el 
desarrollo de habilidades pedagógicas profesionales en el trabajo interdisciplinario.

- Asumir la superación profesional con un enfoque interdisciplinario a partir de la determinación de las necesidades cognoscitivas del claustro.

- Concebir la evaluación con un carácter integral, garantizando la proyección de esta en sus tres modalidades, autoevaluación, coevaluación y heteroevaluación.

\section{CONCLUSIONES}

La necesidad de perfeccionar el modo de actuación profesional desde una perspectiva interdisciplinar, no solo constituye un reto, es una realidad impuesta en la Universidad del Siglo XXI, esto no solo garantiza un acercamiento motivacional-afectivo y cognitivo- instrumental entre docentes y estudiantes, además asegura evitar el distanciamiento entre estas dimensiones, que constituiría un lastre procesal con nefastas consecuencias educativas dentro del proceso. Sólo conjugando la motivación en los diferentes niveles de la personalidad y logrando su efectividad, de forma integral, resultará posible el desarrollo de un modo de actuación profesional de carácter interdisciplinario.

Por tanto, el trabajo del profesional en la Universidad actual debe constituirse en su modo de actuación como una filosofía de trabajo que transite hacia un pensamiento interdisciplinar, de forma tal que pueda asimilar los procesos de forma consciente y menos resistencias, como condición indispensable para su desarrollo y transformación de la diversidad en la que participa.

\section{REFERENCIAS BIBLIOGRÁFICAS}

Addine F. y otros (2007) Didáctica: Teoría y Práctica. La Habana: Pueblo y Educación.

Addine, F. y otros (2002) Principios para la dirección del proceso pedagógico. En Compendio de Pedagogía. La Habana: Pueblo y Educación.

Albert, M. J. (1997) La integración de saberes e interdisciplinariedad, un reto para la formación. En: Colectivo de Autores INTEGRACIÓN DE SABERES E INTERDISCIPLINARIEDAD. Madrid. Universidad Nacional de Educación a Distancia.

Álvarez, M. (2004) La interdisciplinariedad en la enseñanza-aprendizaje de las ciencias. En Âlvarez, M. (comp.) INTERDISCIPLINARIEDAD.
UNA APROXIMACIÓN DESDE LA ENSEÑANZAAPRENDIZAJE DE LAS CIENCIAS. La Habana. Editorial Pueblo y Educación.

Aznar, P. y Angels, M. (2009) La formación de competencias básicas para el desarrollo sostenible: el papel de las universidades, Madrid. REVISTA EDUCACIÓN, número extraordinario, 219-237.

Bolívar, A. (2008) Didáctica y currículum: de la modernidad a la postmodernidad. Achidona (Málaga): Aljibe.

Borbonat, J. (1997) Concepción de la interdisciplinariedad en los distintos niveles educativos. En Colectivo de Autores INTEGRACIÓN DE SABERES E INTERDISCIPLINARIEDAD. Madrid. Universidad Nacional de Educación a Distancia.

Castro, L. (2000) Diccionario de Ciencias de la Educación. Lima. Ceguro Editores.

Colectivo de Autores (1994) Carta de la transdisciplinariedad. Disponible en: www.filosofia. org/cod/c1994tra.htm (Consultado el 7 de Marzo de 2011)

Didrikisson, A. (2010) Una nueva universidad para la sociedad del conocimiento. REVISTA TEMAS, No. 57, 33-41.

Fariñas, G. (2008) Maestro. Para una didáctica del aprender a aprender. La Habana: Félix Varela.

Fiallo, J. (2004) La interdisciplinariedad: un concepto "muy conocido". En Álvarez, M. (comp.) INTERDISCIPLINARIEDAD. UNA APROXIMACIÓN DESDE LA ENSEÑANZA -APRENDIZAJE DE LAS CIENCIAS. La Habana. Editorial Pueblo y Educación. González Rey, F. y Mitjáns, A. (1989) La personalidad. Su educación y desarrollo. La Habana. Pueblo y Educación.

Garcés C., W. (2003) Desarrollo de modo de actuación para el trabajo con sistema de tareas en la formación inicial del profesor de matemática. Tesis Doctoral en Ciencias Pedagógicas. Universidad de Ciencias Pedagógicas. Holguín.

García R., L. (1997, febrero, abril) El modelo de escuela: un problema de la Pedagogía actual. La Habana. En Desafío Escolar: Revista Iberoamericana de Pedagogía. vol. 0. P.2.

González Miriam (2003) La Motivación en el proceso de enseñanza-aprendizaje. Universidad de la Habana, CEPES.

González Muñoz, M. C. (1996) Tratamiento disciplinar versus tratamiento interdisciplinar. REVISTA IBEROAMERICANA DE EDUCACIÓN No.11: 41-45,

González Rey, Fernando. (1989) La personalidad su educación y desarrollo. La Habana. Pueblo y Educación. 
González S., D. J. (1995) Teoría de la Motivación y Práctica Profesional. La Habana. Editorial Pueblo y Educación.

González Serra, Diego j. (1995) Teoría de la Motivación y Práctica Profesional. Cuba. Editorial Pueblo y Educación.

Núñez C. N. (2003) Modelo Interdisciplinario para la formación de actitudes medioambientales. Tesis Doctoral en Ciencias Pedagógicas. Universidad de Ciencias Pedagógicas. Holguín.
Ortiz Emilio (2011) La interdisciplinariedad en las investigaciones educativas. Cuba.

Perea C. (2000). La formación interdisciplinar de los profesores de ciencias: un ejemplo en el proceso de enseñanza aprendizaje de la Física. Tesis Doctoral en Ciencias Pedagógicas. La Habana. 